\title{
La investigación del Internet Addiction Test desde una perspectiva intercultural: España, Estados Unidos y Colombia
}

\section{Internet Addiction Test research through a cross-cultural perspective: Spain, USA and Colombia}

\author{
Tayana Panova*, Xavier Carbonell*, Andres Chamarro**, Diana Ximena Puerta-Cortés***. \\ * FPCEE Blanquerna. Universitat Ramon Llull, Barcelona. España. \\ ** Universitat Autònoma de Barcelona, Cerdanyola del Vallès, Barcelona. España. \\ *** Universidad de Ibagué. Colombia.
}

\section{Resumen}

Los usuarios de Internet frecuentemente experimentan problemas relacionados con su uso de Internet y, aunque la cultura tiene una influencia importante en la forma en que las personas se comunican, en lo que valoran y, por lo tanto, en cómo utilizan Internet, hay escasas publicaciones sobre investigación intercultural del uso problemático de Internet. La herramienta más común para medir dicho uso, el Internet Addiction Test (IAT) se ha utilizado en varios países. En este estudio comparamos los resultados interculturales de las investigaciones más recientes sobre el análisis factorial del IAT. Encontramos que los resultados a menudo se replican en países con dos o más estudios, lo que sugiere que el contexto cultural influye en los comportamientos en Internet. Llevamos a cabo nuestros propios estudios de análisis factorial del IAT en tres países (España, EE. UU. y Colombia) con 1.273 participantes. Al comparar nuestros resultados con los de estudios anteriores, encontramos que nuestros resultados fueron similares a los de estudios anteriores. El hallazgo más notable fue que todos los análisis factoriales de IAT, tanto los nuestros como los anteriores en las mismas regiones, contenían un factor relacionado con la pérdida de control/ problemas de gestión del tiempo y otro factor relacionado con problemas emocionales/psicológicos, lo que sugiere que el control de impulsos y las necesidades emocionales no satisfechas son componentes importantes en el desarrollo del uso problemático de Internet en todo el mundo. La investigación futura sobre el uso problemático de Internet debería centrarse en estos aspectos.

Palabas clave: Adicción a Internet; uso problemático de Internet; intercultural; prueba de adicción a Internet; análisis factorial.

\begin{abstract}
Internet users worldwide often experience problems related to their Internet use, and although culture has an important influence over how people communicate, what they value, and therefore how they use the Internet, little cross-cultural research on the subject of problematic Internet use has been carried out. The Internet Addiction Test (IAT), the most common measurement tool for this purpose, has been used in various countries. In this study we compared and analyzed the cross-cultural results found in the most recent research on the IAT factor analysis. We found that in countries with two or more studies, results are often replicated, suggesting that cultural context influences Internet behaviors. We conducted our own IAT factor analysis studies in three countries - Spain, USA, and Colombia - with a total of 1,273 participants. We compared our results with those from previous studies in the same countries and found similar results. The most notable finding was that all the IAT factor analyses, both ours and the previous ones in the same regions, contained a factor related to loss of control/time management problems and another factor related to emotional/psychological problems, thereby suggesting that impulse control problems and unfulfilled emotional needs are the most important components in the development of problematic Internet use in various countries around the world. Future research on problematic Internet use should focus on these aspects.

Key Words: Internet addiction; problematic Internet use; cross-cultural; Internet addiction test; factor analysis.
\end{abstract}


A unque el uso del Internet es un fenómeno global ha habido pocos estudios sobre el uso problemático de Internet desde una perspectiva intercultural. Esto es una carencia de la literatura que debe abordarse porque las personas de distintas culturas tienen formas muy diferentes de comunicarse, valores y motivaciones y, por tanto, también comportamientos en Internet. Como consecuencia, cuando tienen problemas en relación con su uso de Internet, la naturaleza de estos problemas también difiere según su contexto sociocultural.

Los análisis psicométricos, como el análisis factorial de los cuestionarios sobre la adicción a Internet, muestran resultados diferentes en casi todos los países estudiados. Es más, la mayoría de los estudios comentan que esta variabilidad posiblemente se debe, en parte, a las diferencias culturales. No obstante, muy pocos estudios han analizado la adicción a Internet desde una perspectiva intercultural y se ha prestado poca atención a la cultura en la investigación sobre el uso problemático de Internet en general. Una perspectiva intercultural hacia este ámbito podría ser útil para identificar el impacto de la cultura en los comportamientos problemáticos en Internet. Esto facilitaría el desarrollo de herramientas de evaluación y tratamientos personalizados para los usuarios de Internet con problemas en distintas poblaciones.

\section{El uso problemático de Internet y la adicción a Internet}

El uso problemático de Internet (UPI) es un problema importante como objeto de estudio, dado que la mitad de la población mundial usa Internet con regularidad y la popularización del teléfono inteligente ha facilitado y aumentado la frecuencia del acceso a Internet (Stevens, 2018). Estudios han mostrado que la adicción a Internet (AI) está asociada con trastornos como ansiedad (Ho et al., 2014; Lee y Stapinski, 2012; Younes et al., 2016), depresión (Orsala, Orsalb, Unsalc y Ozalp, 2013; Younes et al., 2016), estrés (Pedrero-Pérez et al., 2018; Samaha y Hawi, 2016; Younes et al., 2016), baja autoestima (Bahrainian, Alizadeh, Raeisoon, Hashemi y Khazaee, 2014; Bozoglan, Demirer y Sahin, 2013), aislamiento (Bozoglan, Demirer y Sahin, 2013; Yao y Zhong, 2014), insomnio (Chen y Gau, 2016; Younes et al., 2016), tendencias suicidas (Lin et al., 2014), impulsividad (Lee, Choi, Shin, Lee, Jung y Kwon, 2012), abuso de sustancias (Ho et al., 2014; Lee, Han, Kim y Renshaw, 2015) y TDAH (Ho et al., 2014; Weinstein, Yaacov, Manning, Danon y Weizman, 2015), entre otros. Dicho esto, aún se debate si el uso problemático de Internet se puede etiquetar como una adicción (Kardefelt-Winther, 2014; Sánchez-Carbonell, Beranuy, Castellana y Chamarro, 2008; Starcevic, 2013; Widyanto y Griffiths, 2006), y la adicción a Internet quedó excluida del DSM-V (Petry y O’Brien, 2013) y del CIE-11 (Bobes, Flórez, Seijo y Bobes, 2019), aunque se han incluido algunas activida- des problemáticas específicas en línea, como los juegos de apuestas y los videojuegos. La reticencia a confirmar de manera definitiva un trastorno de adicción a Internet se debe a cuestiones relacionadas con su desarrollo teórico, metodología y conceptualización entre los estudios, y porque los niveles de severidad de los problemas asociados a la «adicción» a Internet no suelen ser comparables con la severidad de los problemas causados por otras adicciones confirmadas.

El enfoque sobre el marco de la adicción puede haber contribuido hacia los problemas relacionados con el diagnóstico y tratamiento del uso problemático de Internet porque la herramienta principal para diagnosticarla, el Internet Addiction Test (IAT), tiene una validez estructural inestable. El IAT fue desarrollado por Young (1998), a partir de los criterios del DSM-IV (American Psychiatric Association, 1994) para juego patológico, y ha sido el instrumento más usado a nivel mundial para estudiar el uso problemático de Internet. Su diseño tiene una estructura unidimensional, pero se ha encontrado que tiene entre uno y seis factores (Laconi, Rodgers y Chabrol, 2014). Aunque su fiabilidad es consistentemente fuerte (Laconi, Rodgers y Chabrol, 2014; Panayides y Walker, 2012), su estructura factorial difiere en casi todos los estudios, por lo que es difícil identificar qué componentes del uso problemático de Internet son más relevantes para abordar en el diagnóstico y tratamiento. Muchos de los estudios en la literatura sobre el análisis factorial del IAT mencionan el posible papel de la cultura sobre las diferencias psicométricas halladas entre estudios; no obstante, hay pocos estudios sobre el IAT desde una perspectiva intercultural.

\section{Cultura}

Aunque ha habido pocos estudios sobre adicción al Internet/uso problemático de Internet (UPI) desde un enfoque cultural (Lopez-Fernandez, 2015), aquellos que existen hallaron diferencias culturales interesantes en el uso de Internet.

Durkee et al. (2012) estudiaron el uso patológico de Internet en Alemania, Austria, Eslovenia, España, Estonia, Francia, Hungría, Irlanda, Israel, Italia, Rumanía, y Suecia; Israel tuvo la tasa más elevada de uso inadaptado del Internet $(18,2 \%)$ y uso patológico de Internet $(11,8 \%)$, e Italia las tasas más bajas $(8,8 \%$ y $1,2 \%)$. Al comparar zonas metropolitanas y micropolitanas encontraron que los adolescentes que viven en las primeras tenían un riesgo mayor de UPI. Destacaron la importancia de este hallazgo e indicaron que la diferencia significativa entre las culturas metropolitana vs. micropolitana merece más estudio.

Tsitsika et al. (2014) encontraron que las tasas de prevalencia de comportamiento adictivo al Internet fueron más elevadas en los países del sur, este, y centro de Europa, y más bajas en los del norte de Europa. Específicamente, encontraron que los países con los comportamientos en 
Internet más y menos disfuncionales, respectivamente, fueron España, con una tasa de $23 \%$, e Islandia con $8 \%$. Estos resultados contradicen los de otro estudio intercultural sobre el uso de Internet realizado por Laconi et al. (2018) que comparó el uso problemático de Internet en Alemania, España, Francia, Grecia, Hungría, Inglaterra, Italia, Polonia y Turquía; encontró que la muestra española tenía una de las tasas más bajas de UPI.

Seabra et al. (2017) encontraron una paradoja interesante en su comparación de usuarios de Internet portugueses y brasileños. Esperaron encontrar un mayor uso problemático entre los usuarios portugueses, dado su mayor número de usuarios de Internet per cápita y acceso más fácil al Internet que los brasileños. No obstante, encontraron que los usuarios brasileños tenían niveles más altos de adicción a Internet, demostrando así que la facilidad de acceso y la prevalencia de uso de un país no son suficientes para predecir el uso problemático de Internet.

Para facilitar una comparación intercultural de los análisis factoriales del IAT a nivel mundial, organizamos los resultados más recientes de los metaanálisis sobre el análisis factorial del IAT (Moon et al., 2018) por región geográfica e identificamos similitudes/diferencias interesantes (Tabla 1). Un aspecto del estudio que consideramos interesante destacar fue las diferentes maneras en que los equipos de investigadores etiquetaron los factores que identificaron. No hay un solo estándar para nombrar factores, por lo que los nombres de los factores seleccionados por cada equipo indican cómo resumieron la combinación de ítems dentro de ese factor. Esto nos ofrece conocimientos sobre cómo los equipos de distintos países interpretan los factores y cuáles aspectos de los mismos consideran más importantes.

\section{Comparación de los análisis factoriales del IAT a nivel mundial}

Asia y Europa tenían el número más elevado de estudios sobre los análisis factoriales del IAT, así que comparamos sus factores para identificar similitudes y/o diferencias, en su caso. Debemos señalar un par de cosas respecto de los nombres de los factores en sí. Primero, Asia fue el único continente en el cual se usó la palabra «aislamiento» (en el sentido de aislarse de los demás) para nombrar los factores y el $50 \%$ de los estudios mencionó «negligencia del trabajo/las obligaciones», mientras que solo un estudio en todos los demás artículos a nivel mundial mencionó este tipo de constructo (Tsimtsiou et al., 2014). Desde una perspectiva cultural, estas diferencias pueden deberse a la naturaleza colectiva de la mayoría de las comunidades asiáticas. Tener un papel activo en la sociedad es muy importante y altamente valorado, por lo que aislarse del grupo o descuidar el rol que uno tiene en la comunidad se considera señal de un problema. A su vez, casi un $60 \%$ de los estudios europeos usó la palabra «emoción/estado de ánimo» para nombrar los factores, mientras que no apareció en ningún estudio asiático. Esto puede deberse a que los países europeos, en comparación con los asiáticos, tienden a ser más individualistas, prestan más atención a las experiencias personales e internas de las personas (Hofstede, 1983). Faltan datos para poder llegar a conclusiones sobre esto, por lo que recomendamos que se hagan más estudios para explorar en profundidad estas posibles diferencias culturales.

Debido a la gran variabilidad en los resultados entre estudios para llegar a conclusiones fiables respecto de regiones geográficas más amplias, y porque es poco sensato basarse en un único estudio para representar fiablemente a una población, en su lugar examinamos los países con dos o más estudios del análisis factoriales del IAT para ver si los resultados se replicaban, lo que consolidaría la teoría que el contexto cultural influye sobre los comportamientos en Internet. Identificamos tres países del metaanálisis en los cuales se habían hecho dos o más estudios -Corea del Sur, Italia y Turquía- y comparamos sus resultados para ver cómo de estrechamente ser replicaban sus resultados.

Los resultados fueron similares en los estudios en Corea del Sur: ambos estudios tuvieron un factor primario centrado en la gestión del tiempo, con casi todos los ítems del Factor 1 de Sung, Shin y Cho (2014) incluidos, a su vez, en el Factor 1 de Lee et al. (2013). Ambos estudios también tenían un factor de aislamiento, con todos los ítems del factor de aislamiento de Lee et al. incluidos en el factor de aislamiento de Sung et al. Los estudios tenían diferentes muestras: la de Sung se compuso de participantes adolescentes entre las edades de 13-15 años y la de Lee se compuso de estudiantes universitarios. Esta diferencia de edad puede ser un factor contribuyente a los diferentes hallazgos entre los estudios: Sung et al. encontraron cuatro factores y Lee et al. encontraron tres.

Los tres estudios de Italia (Faraci, Craparo, Messina y Severino, 2013; Fioravanti y Casale, 2015; Servidio, 2017) mostraron prácticamente los mismos resultados. Cada estudio estuvo compuesto de estudiantes universitarios (Fiorvanti y Casale usaron estudiantes universitarios y de secundaria), y cada uno tenía una estructura de dos factores con factores primarios y secundarios con casi los mismos ítems entre todos los estudios; un factor se relacionó con los problemas emocionales/psicológicos y el otro con la pérdida del control sobre el tiempo y la interferencia con la vida cotidiana.

Los resultados de Turquía (Boysan et al., 2017; Kaya, Delen y Young, 2016) fueron diferentes: aunque ambos estudios explicaron casi el mismo porcentaje de variación (46\% en Kaya et al. y 45\% en Boysan et al.), Boysan et al. hallaron una estructura unidimensional mientras que Kaya et al. identificaron cuatro factores independientes. Dado que las muestras fueron muy similares en términos culturales y demográficos, las diferencias podrían atribuirse al hecho de que se usaron diferentes análisis estadísticos. 
Tabla 1. Tabla de estudios internacionales sobre el análisis factorial del IAT.

\begin{tabular}{|c|c|c|c|c|}
\hline Población estudiada & $\begin{array}{c}\text { N. } .9 \text { de } \\
\text { participantes }\end{array}$ & $\begin{array}{l}\text { N.. } . \text { de } \\
\text { factores }\end{array}$ & $\begin{array}{l}\text { Varianza } \\
\text { total (\%) }\end{array}$ & $\begin{array}{l}\text { Nombre de los factores } \\
\text { (y número de ítems) }\end{array}$ \\
\hline
\end{tabular}

1. Malasia: Guan et al. (2015)

3. Bangladesh: Karim et al. (2014)

Estudiantes universitarios

172

4. Corea del Sur: Sung et al. (2014)

Estudiantes de secundaria

1.722

Estudiantes universitarios

279

Estudiantes de bachillerato y

6. China: Lai et al. (2013)

de secundaria

162
2. India: Dhir et al. (2015)

5

64,0

1. Gestión del tiempo (6), comportamientos de aislamiento (8), negligencia de las obligaciones laborales (4).

2. Pérdida de control (8), negligencia de las obligaciones (7), uso problemático (2), perturbación de las relaciones sociales (2), priorización del correo electrónico (1)

3. - .

4. Negligencia de las obligaciones (6), dependencia de estar en línea (4), fantasías virtuales (5), privacidad y autodefensa (3).

5. Uso excesivo de Internet (9), dependencia (5), aislamiento (3), evitación de la realidad (3).

6. Aislamiento y problemas sociales (9), gestión del tiempo y rendimiento (6), sustitución de la realidad (3).

Europa

1. Italia: Servidio (2017)

2. Turquía: Boysan et al. (2016)

3. Turquía: Kaya et al. (2016)

4. Polonia: Hawi et al. (2015)

5. Italia: Fioravanti et al. (2015)

Estudiantes universitarios

Estudiantes universitarios

Estudiantes universitarios

453

Estudiantes universitarios

407

Estudiantes con edades entre

14-26 años

1.245

840

6. España: Fernandez-Villa et al. (2015) Estudiantes universitarios

Estudiantes universitarios de

7. Grecia: Tsimtsiou et al. (2014)

medicina

8. Portugal: Pontes et al. (2014)

Estudiantes de bachillerato y

universitarios

Estudiantes universitarios

Estudiantes universitarios

Estudiantes universitarios de psicología

Población general (usuarios

de Internet)

3

9. Italia: Faraci et al. (2013)

10. Portugal: Conti et al. (2012)

11. Alemania: Barke et al. (2012)

12. Reino Unido: Widyanto et al. (2011)

$851 \quad 2 \quad 55,0$

41,0 1. Conflictos interpersonales, emocionales y obsesivos como resultado del uso de Internet (11), gestión del tiempo en línea y negligencia del bienestar (7).

2. - .

3. Estado de ánimo (8), relaciones (4), responsabilidades (5), duración (3).

4. Trastorno mental (11), trastorno de gestión de tiempo (9).

5. Obsesión emocional y cognitivo con el Internet y consecuencias sociales (11), pérdida de control e interferencia con las obligaciones diarias (9).

$151 \quad 3 \quad 55,3 \quad 6$. Inversión emocional (11), gestión del tiempo y rendimiento (8).

7. Conflicto psicológico/emocional (10), gestión del tiempo (6), negligencia de las obligaciones laborales (4).

42,2 9. Obsesión emocional y cognitiva con el Internet (11), pérdida de control e interferencia con las obligaciones diarias (7).

$$
77
$$

1,041 online $y$ 841 offline

\section{225}

10.-.

46,7, 11. Obsesión (12), pérdida de control online (8). (8).

12. Conflicto emocional/psicológico (9), problemas con la gestión de tiempo (5), cambio del estado de ánimo (6).

56,3
12. 3

Norteamérica

1. Canadá: Watters et al. (2013)

Estudiantes de bachillerato

1.948

2. EE. UU.: Jelenchick et al. (2012)

Estudiantes universitarios con edades entre 18-20 años

215

1. -

2. Uso dependiente (12), uso excesivo

Sudamérica

1. Colombia: Puerta-Cortés et al.

Población general (usuarios

1.117

1. Consecuencias por el uso de Internet (2012) de Internet)

47,8

(7), dimensión cognitiva y emocional

África

$-$

Oriente Medio Asia

1. Pakistán: Waqas et al. (2018)

Estudiantes universitarios

(medicina y odontología).

522

2. Persia: Mohammadsalehi et al. (2015)

Estudiantes universitarios (ciencias médicas)

3. Jordania: Ahmad et al. (2015)

4. Líbano: Hawi (2013)

Estudiantes universitarios

Estudiantes de bachillerato y

de secundaria

$-$

$\begin{array}{lllll}- & - & - & -\end{array}$
(10), control del tiempo (3). 


\section{Hipótesis}

Dado que los dos estudios de replicación de los análisis factoriales del IAT dentro del mismo país mostraron resultados muy similares (Corea e Italia) y un estudio de replicación no obtuvo resultados similares al estudio original (Turquía), quisimos explorar aún más si los resultados de los estudios sobre los análisis factoriales del IAT podrían replicarse en un mismo país. Dado que los análisis factoriales del IAT son tan diferentes entre países, con entre uno y cinco factores, nuestra hipótesis fue que, si los análisis factoriales del IAT en un mismo país mostraban resultados muy similares, esto apoyaría la hipótesis que las diferencias culturales influyen sobre los comportamientos del IAT. Seleccionamos tres países en los cuales los análisis factoriales del IAT se habían realizado anteriormente y que eran considerablemente diversos en términos de región geográfica, características socioeconómicas y cultura: EE. UU. (región del Medio Oeste), España (Barcelona) y Colombia (Ibagué).

\section{¿Por qué EE. UU., España y Colombia?}

Cada uno de los países de este estudio es especial respecto de sus estudios sobre el uso del Internet; EE. UU. es uno de los tres principales países mundiales respecto de la penetración del Internet, Colombia tiene tasas de uso de Internet representativas de toda Sudamérica porque sus tasas de penetración de Internet son iguales que el nivel medio de todos los países latinoamericanos (Comisión Económica para América Latina y el Caribe, 2017) y España es representativa del uso de Internet en Europa porque sus tasas de penetración de Internet son iguales que el nivel medio de todos los países europeos (Netherlands Leads Europe 2018). Realizamos un análisis factorial del IAT para una muestra de estudiantes universitarios para comprobar si los resultados serían similares a los resultados de los estudios de análisis factorial realizados anteriormente en ese país. Usamos estudiantes universitarios porque los estudios anteriores también lo hicieron (el estudio colombiano se había hecho con muestras de la población general; no obstante, la edad media fue 20,93 años, y la mayoría de los participantes tenían una edad universitaria).

\section{Métodos}

\section{Participantes}

Los participantes fueron 1.516 estudiantes de tres universidades, una en cada país, que rellenaron un cuestionario en línea. Tras eliminar los cuestionarios incompletos, quedaron 451 de EE. UU., 467 de España y 355 de Colombia. Las mujeres representaron el 64,9\% de la muestra estadounidense, el 79,2\% de la española y el $64,2 \%$ de la colombiana. Las edades medias de los estudiantes fueron: de los americanos 19,59 años $(S D=1,43$; rango 18-30), de los españoles 21,45 años $(S D=2,41$; rango $18-30)$ y de los colombianos 19,95 años ( $S D=2,00$; rango 18-30). Los participantes españoles tenían una edad ligeramente mayor que los otros dos grupos $(F=111,05 ; p<, 001)$.

\section{Medidas}

Internet Addiction Test (Young, 1998): un cuestionario autoinformado de 20 ítems, a partir de los criterios del DSMIV para juego patológico. Se pide a los participantes que puntúen los ítems en una escala tipo Likert de cinco puntos acerca del grado en el cual su uso de Internet afecta su rutina diaria, vida social, productividad, patrones de sueño y sentimientos. La puntuación mínima es de 20 y la máxima es de 100. Cuanto mayor la puntuación, mayores los problemas que causa el uso de Internet. Young sugirió que una puntuación entre 20 y 39 es lo típico de un usuario del Internet que no tiene problema alguno con su uso del Internet. Una puntuación entre 40 y 69 significa problemas frecuentes debido al uso del Internet. Por último, una puntuación entre 70 y 100 significa que el Internet causa problemas significativos para el usuario. El IAT fue disenado como un instrumento unidimensional; no obstante, estudios posteriores han hallado entre uno y seis factores (véase Moon et al. 2018). En el uso en línea, la fiabilidad varía entre 83 y 91 (Korkeila, Karlaas, Jääskeläinen, Vahlberg y Taiminen, 2010; Barke, Nyenhuis y Kröner-Herwig, 2012; Jelenchik, Becker y Moreno, 2012). Nuestro estudio tuvo una fiabilidad (alfa de Cronbach) de 91.

\section{Procedimiento}

En las universidades en España y Colombia, los investigadores enviaron un correo electrónico a los estudiantes invitándoles a participar en el estudio, y en los EE. UU. los participantes fueron reclutados a través de la página web Subject Pool de Universidad de Illinois (usada por los estudiantes para encontrar y participar en proyectos de investigación). Los participantes que decidieron participar hicieron clic en el enlace que se les envió desde el mismo correo electrónico o desde la página web Subject Pool (EE. UU.) y fueron redirigidos a los cuestionarios en línea en el alojamiento web de Qualtrics. Al acceder al cuestionario, se le presentó un documento explicando el estudio y solicitando su consentimiento informado para continuar. No se recopilaron datos personales de los participantes, se codificaron sus respuestas como un conjunto de números y letras aleatorios y no se registraron sus números IP. Algunos de los datos recopilados no estaban relacionados con el enfoque sobre el Internet del estudio actual, por lo que no se presentan aquí.

\section{Análisis de datos}

Para el análisis de los componentes principales, usamos la rotación Varimax de extracción de datos. Antes del análisis factorial exploratorio, revisamos los datos para asegurar que los ítems tenían una correlación significativa, con la 
prueba de esfericidad de Bartlett. Además, para evaluar si los ítems compartían una varianza suficiente para justificar la extracción factorial, usamos la medida de adecuación muestral de KMO. Las cargas factoriales que resultaron de la rotación Varimax se evaluaron con un umbral de ,40. Cuando un ítem tenía cargas en más de un factor, se juntó con el factor con la carga más alta, a no ser que hubiera un motivo convincente para juntarlo a otro factor con el fin de mejorar la interpretación de los factores. Verificamos la estructura factorial del IAT que surgió del análisis factorial exploratorio usando un análisis factorial confirmatorio (seleccionamos como procedimiento de estimación el AFC de mínimos cuadrados, aplicable cuando los datos incumplen el supuesto de una distribución normal multivariante). El ajuste del modelo se evaluó con base al índice de ajuste comparativo (CFI), el índice de Tucker-Lewis (TLI), el error cuadrático medio de aproximación (RMSEA) y la raíz cuadrada media residual estandarizada (SRMR). CFI y TLI $>, 90$, RMSEA $<, 08$ and SRMR $<, 1$ típicamente reflejan un ajuste aceptable y CFI y TLI $>, 95$, RMSEA <,06 y SRMR $<, 08$ indican un ajuste excelente (Brown, 2006). También hicimos análisis descriptivos y correlacionales. Para comprobar las diferencias entre país y sexo, hicimos un análisis de varianza bifactorial (sexo por país) mediante el procedimiento de modelo lineal general. En el caso de efectos principales significativos, calculamos comparaciones post hoc (mediante el ajuste para comparaciones múltiples de Bonferroni). Usamos la versión 19 del software SPSS para calcular las estadísticas descriptivas, el modelo lineal general y el análisis factorial exploratorio (AFE). Usamos el programa EQS 6.1 (Bentler, 2006) para el análisis factorial confirmatorio (AFC).

\section{Resultados}

\section{Análisis descriptivo}

De la muestra íntegra, el $72 \%$ de los encuestados obtuvo puntuaciones entre 20 y 39, lo que significa que no tenían problemas con su uso del Internet. El $27 \%$ obtuvo puntuaciones entre 40 y 69 , lo que significa que tuvieron problemas frecuentes debido al uso del Internet, y el $1 \%$ obtuvo puntuaciones entre 70 y 100, para los cuales el Internet puede ser un problema significativo. Respecto de las diferencias entre países, los participantes españoles obtuvieron puntuaciones más bajas en el IAT ( $M=33,50 ; S D$ $=9,44)$ que los participantes estadounidenses $(M=36,82$; $S D=10,82)$ y los participantes colombianos $(M=36,70 ; S D$ $=11,05)$. Las diferencias fueron estadísticamente significativas $(F=12,55 ; p=, 000)$.

\section{Análisis factorial}

La medida de adecuación muestral de KMO fue ,94 y la prueba de esfericidad de Bartlett $(\chi=9490,9)$ fue significativa $(P=, 000)$, lo que indica que los ítems del IAT fueron adecuados para un análisis factorial. Para ambos EE. UU. y España, este criterio resultó en una solución de tres factores, mientras que en el caso de Colombia, hubieron dos factores subyacentes. La Tabla 1 muestra las cargas factoriales de los ítems para EE. UU., España y Colombia, respectivamente.

Para EE. UU., los tres factores explicaron el 51,91\% de la varianza (Tabla 1). El factor 1 (doce ítems) representó el $25,65 \%$ de la varianza y aparentemente midió el conflicto psicológico. El factor 2 (cinco ítems) representó el $16,67 \%$ de la varianza y aparentemente midió la incapacidad para controlar el uso. El factor 3 (tres ítems) representó el 9,66\% de la varianza y aparentemente midió las disfunciones sociales y laborales. Este modelo tuvo un ajuste excelente (CFI =,985; TLI =,983, RMSEA =,027; SRMR $=, 042)$. Para España, los tres factores explicaron el 46,68\% de la varianza. El factor 1 (7 ítems) representó el 18,16\% de la varianza y midió las disfunciones sociales y laborales y las dificultades con la gestión del tiempo. El factor 2 (8 ítems) representó el 15,55\% de la varianza y midió los conflictos psicológicos relacionados con el uso de Internet. El factor 3 (4 ítems) represento el 13,14\% de la varianza y midió la reacción afectiva. El ítem 14 no presentó carga sobre ningún otro factor. Este modelo tuvo un ajuste excelente $(\mathrm{CFI}=, 989$; TLI =,987, RMSEA =,023; SRMR =,040). Para Colombia, los dos factores explicaron el $54,7 \%$ de la varianza. El factor 1 (11 ítems) representó el 30,72\% de la varianza y midió los conflictos psicológicos. El factor 2 (8 ítems) representó el 23,97\% de la varianza y midió la incapacidad para controlar el uso. El ítem 7 no presentó carga sobre ningún otro factor. Este modelo tuvo un ajuste aceptable $(\mathrm{CFI}=, 978$; TLI =,975, RMSEA =,033; SRMR =,049).

\section{Resumen}

Para cada país, presentamos abajo los nombres tanto simplificados como completos de los factores:

- EE. UU.:

- Factor 1: Necesidad emocional (satisfacción de necesidades emocionales y dependencia): $3,4,5,9,10,11$, 12, 13, 15, 18, 19, 20.

- Factor 2: Pérdida de control (incapacidad para controlar el uso y descuido de actividades importantes): $1,2,14,16,17$.

- Factor 3: Negligencia de las obligaciones (negligencia de las obligaciones a favor de Internet): 6, 7, 8 .

\section{- España:}

- Factor 1: Pérdida de control (incapacidad para controlar el uso y negligencia de las obligaciones) 1, 2, 6 , $7,8,16,17$.

- Factor 2: Necesidad emocional (satisfacción de necesidades emocionales) 3, 4, 9, 10, 13, 18, 19, 20.

- Factor 3: Dependencia: 5, 11, 12, 15.

\section{- Colombia:}


- Factor 1: Necesidad emocional (satisfacción de necesidades emocionales y dependencia): $3,4,9,10,11$, 12, 13, 15, 18, 19, 20.

- Factor 2: Pérdida de control (incapacidad para controlar el uso y negligencia de las obligaciones): 1, 2, $5,6,8,14,16,17$.

Tabla 2. Análisis factorial del IAT en los tres países.

\begin{tabular}{|c|c|c|c|c|c|c|c|c|}
\hline & \multicolumn{3}{|c|}{ EE. UU. } & \multicolumn{3}{|c|}{ España } & \multicolumn{2}{|c|}{ Colombia } \\
\hline & 1 & 2 & 3 & 1 & 2 & 3 & 1 & 2 \\
\hline P1 &,- 013 & ,709 & ,342 & ,584 &,- 050 & ,398 & ,039 & ,767 \\
\hline P2 & ,182 &, 569 & ,380 &, 725 & ,170 &, 260 & ,299 & ,731 \\
\hline P3 &, 561 &,- 068 &, 355 & ,022 & ,435 &,- 001 &, 569 & 131 \\
\hline P4 &, 522 & ,090 &, 080 & ,193 & ,474 &, 120 & ,629 & ,303 \\
\hline P5 & ,626 & ,146 & ,118 &, 250 &, 264 & ,464 &, 277 & ,673 \\
\hline P6 & ,301 & ,336 &, 600 & ,707 & ,278 & ,081 &, 540 &, 555 \\
\hline P7 &,- 087 & ,167 & ,661 & ,466 &,- 035 & ,260 & ,225 & ,253 \\
\hline P8 &, 347 & ,232 &, 657 &, 725 & ,247 & ,006 & ,482 & ,612 \\
\hline P9 & ,595 & ,139 & ,204 & ,207 & ,615 & ,177 & ,651 & ,349 \\
\hline P10 & ,407 & ,349 & ,346 & ,165 &, 502 & ,286 & ,679 & ,356 \\
\hline P11 &, 588 & ,403 & ,029 & ,245 & 190 & ,649 & ,667 & ,368 \\
\hline P12 &, 566 &, 425 &,- 050 & ,095 & ,083 & ,701 & ,626 & ,436 \\
\hline P13 & ,697 & ,084 &, 016 & ,173 &, 538 & ,408 & ,713 & ,223 \\
\hline P14 & ,246 & ,647 & ,104 & ,357 & ,353 & ,305 & ,355 & ,587 \\
\hline P15 & ,732 & ,389 &,- 024 & ,215 & ,249 & ,715 & ,700 & ,377 \\
\hline P16 & ,154 & ,806 & ,102 & ,680 &, 210 &, 251 & ,283 & ,707 \\
\hline P17 & ,254 & ,688 & ,203 & ,655 & ,330 & ,112 &, 374 & ,651 \\
\hline P18 & ,709 & ,194 & ,152 & ,407 &, 514 & ,123 & ,571 & ,488 \\
\hline P19 & ,669 &, 225 & ,192 & ,100 &, 765 & ,099 & ,796 & ,211 \\
\hline P20 & ,772 & ,093 &, 084 & ,114 &, 554 & ,450 & ,812 & ,222 \\
\hline
\end{tabular}

\section{Discusión}

\section{Comparación de los análisis factoriales del IAT}

Encontramos muchas similitudes cuando comparamos nuestros hallazgos con los análisis factoriales del IAT en los mismos países. Encontramos tres factores en la muestra de EE. UU.: 1) Satisfacción de necesidades emocionales y dependencia, 2) Incapacidad para controlar el uso y descuido de actividades importantes, y 3) Negligencia de las obligaciones a favor de Internet. Los análisis factoriales del IAT en los EE. UU. (Jelenchick, Becker y Moreno, 2012) hallaron dos factores titulados 1) «Uso dependiente», y 2) «Uso excesivo». El comparar nuestros hallazgos con los suyos, encontramos que ambos estudios tuvieron un factor 1 idéntico y un factor 2 muy similar (todos los ítems del factor 2 de nuestro estudio fueron incluidos en el factor 2 de Jelenchick). La diferencia principal fue que los tres ítems adicionales en el factor 2 de Jelenchick aparecieron como un factor independiente en nuestro estudio: aquellos ítems relacionados con la negligencia de las obligaciones laborales o académicas a favor de Internet. Los ítems compartidos en el factor de Necesidad emocional están relacionados con la dependencia de Internet para obtener afecto positivo y preferir el Internet sobre la realidad. Los ítems compartidos en el factor de Pérdida de control están relacionados con la incapacidad para controlar el tiempo que uno está en línea y priorizar el tiempo dedicado al Internet sobre otras tareas.

También encontramos tres factores en nuestra muestra de España: 1) Incapacidad para controlar el uso y negligencia de las obligaciones, 2) Satisfacción de necesidades emocionales, y 3) Dependencia. Los análisis factoriales del IAT anteriores en España (Fernández-Villa et al., 2015) encontraron dos factores llamados: 1) «Inversión emocional», y 2) «Rendimiento y gestión del tiempo». De nuevo, encontramos similitudes cuando comparamos nuestros hallazgos con los suyos. Casi todos los ítems en nuestro factor 2 fueron incluidos en el factor 1 de Fernández-Villa et al., aunque su factor 1 tuvo unos 4 ítems adicionales, 3 de los cuales componían nuestro factor 3 . Además, nuestro factor 1 fue casi idéntico al factor 2 de Fernández-Villa et al. Aunque ambos factores ocupan posiciones distintas en los dos estudios, es importante señalar sus similitudes; como fue el caso con la muestra estadounidense, un factor compartido entre ambos estudios se centró en los problemas psicológicos/emocionales y el otro factor compartido se centró en los problemas relacionados con la pérdida de control/ gestión del tiempo. Los ítems compartidos en el factor de Necesidad emocional principalmente estaban relacionados con la dependencia de Internet para obtener afecto positivo. Los ítems compartidos en el factor de Pérdida de control estaban relacionados con la incapacidad para controlar el tiempo y la negligencia de las obligaciones a favor de Internet.

Encontramos dos factores en nuestra muestra de Colombia: 1) Satisfacción de necesidades emocionales y dependencia, y 2) Incapacidad para controlar el uso y negligencia de las obligaciones. Los análisis factoriales anteriores del IAT en Colombia (Puerta-Cortés, Carbonell y Chamarro, 2012) hallaron tres factores, titulados: 1) «Consecuencias del uso de Internet», 2) «Dimensión cognitiva-emocional», y 3) «Control del tiempo». De nuevo, encontramos muchas similitudes cuando comparamos nuestros hallazgos con los suyos. El factor 2 de Puerta-Cortés y nuestro factor 1 son casi idénticos, con la diferencia solo en un ítem. El factor 1 de Puerta-Cortés también comparte una mayoría de sus ítems con nuestro factor 2. Los ítems compartidos en el factor de Necesidad emocional fueron casi los mismos que los de la muestra de EE. UU., relacionados con la depen- 
dencia de Internet para obtener afecto positivo y preferir el Internet sobre la realidad. Los ítems compartidos en el factor de Pérdida de control estaban relacionados con el exceso de tiempo empleado en línea y la negligencia de las obligaciones a favor de Internet.

Dado que todas nuestras replicaciones de los análisis factoriales del IAT mostraron resultados muy similares a los estudios anteriores realizados en esos países, nuestra hipótesis fue respaldada porque la replicación en un mismo país de los análisis factoriales del IAT son similares, mientras que los análisis entre países alrededor del mundo suelen ser diferentes; por tanto, parece que la cultura influye sobre cómo se expresa el uso problemático de Internet. Consecuentemente, debemos tener la cultura en mente al investigar el uso del Internet y debemos hacer más estudios sobre cómo la cultura influye los comportamientos en Internet. No obstante, también es importante destacar que en los tres países que estudiamos, encontramos presentes las dos mismas categorías básicas, lo que indica que un patrón universal subyace el uso problemático de Internet.

\section{Los factores compartidos y las implicaciones para la investigación sobre adicción a Internet}

Todas nuestras muestras, y los estudios con los cuales las comparamos, tenían uno de sus dos primeros factores enfocados en problemas relacionados con la pérdida de control/gestión del tiempo, y el otro de los dos primeros factores enfocados en los problemas emocionales/psicológicos, a pesar de que estos factores se expresaron de manera algo diferente entre países. Este hallazgo confirma los resultados del metaanálisis de Moon et al. (2018) que determinó que cuando se consideran solo los estudios que se adhieren estrictamente a las directrices del análisis factorial, es más probable que el IAT tenga uno o dos factores reales. Los seis estudios compartieron dos ítems en el factor de Pérdida de control: las preguntas 1 y 2 . Los seis estudios compartieron seis ítems del factor de Necesidad emocional: las preguntas 3, 9, 10, 13, 19 y 20.

Todos los estudios italianos compartieron estos dos mismos factores, además de los coreanos como a pesar del hecho de que Lee et al. (2012) encontraron cuatro factores y Sung et al. (2014) encontraron tres. Por tanto, podemos concluir que, aunque hay diferencias en los hallazgos de los análisis factoriales del IAT internacionalmente, con los factores variando entre 1-5 en los metaanálisis más reciente (Moon et al., 2018), suelen emerger dos factores primarios: uno relacionado con problemas emocionales/dependencia, y el otro relacionado con problemas de pérdida de control/gestión del tiempo respecto del Internet. Los futuros esfuerzos diagnósticos y de tratamiento deberían centrarse en estos dos factores en la ausencia de información más detallada sobre la población específica objeto del estudio. Es más, dada la plausibilidad de una estructura de dos factores, los estudios futuros con análisis estadísticos modernos, como el Modelo Exploratorio de Ecuaciones Estructurales (MEEE) (ESEM; Asparouhov y Muthen, 2009) que permite la posibilidad de cargas cruzadas (i.e., que un ítem puede ser indicativo de dos factores latentes), deberían usarse para probar la estructura y la invarianza intercultural del IAT.

Respecto del tema de la cuestionada existencia de la Adicción al Internet como trastorno, juzgando desde los dos factores más comunes identificados en el IAT, por lo visto hay dos componentes primarios subyacentes del uso problemático de Internet: problemas para controlar los impulsos y la presencia de necesidades emocionales no satisfechas que buscan su satisfacción mediante el Internet. Parece ser que la comorbilidad de estos dos componentes se manifiesta como un uso problemático de Internet en la moderna edad de la tecnología. Con esto en mente, puede ser más ventajoso desde la perspectiva del diagnóstico y el tratamiento centrarse en dos componentes cognitivo-emocionales en lugar de en la «Adicción al Internet» como constructo único, que todavía es un concepto algo difuso con varias interpretaciones.

Como han mostrado los análisis factoriales realizados internacionalmente, aunque el IAT puede haberse diseñado como una medida unidimensional, esta unidimensionalidad no se ha demostrado. Por tanto, quizás la Adicción al Internet no debe considerarse un constructo único, sino que los comportamientos en Internet problemáticos deberían considerase como una expresión en la actualidad de una combinación de trastornos cognitivo-emocionales (Starcevic, 2010) que antes de la existencia de Internet se manifestaban de otras maneras, pero que mantienen los mismos constructos básicos, independientemente del mismo. Porque la «Adicción al Internet» ha sido difícil de describir en términos psicométricos debido a la inestabilidad de las medidas diagnósticas, y porque el uso problemático de Internet se presenta de maneras diferentes en personas alrededor del mundo, y porque hay pocos casos clínicos de adictos al Internet, quizás la base es insuficiente para diagnosticar la adicción al Internet en estos momentos. Sería más beneficioso para la prevención, el diagnóstico y el tratamiento de aquellas personas que sufren del uso problemático de Internet si más estudios explorasen cuáles perfiles cognitivo-emocionales son susceptibles a tener problemas con el uso de Internet y por qué, cuáles motivaciones son las que les impulsan a ese uso problemático, y cuáles beneficios obtienen al usar Internet que son tan poderosos como refuerzos que priorizan ese uso sobre otros aspectos de la vida cotidiana (Grande, Martínez y Fernández, 2019; Kardefelt-Winther, 2014).

\section{Limitaciones}

Este estudio no está exento de limitaciones. Primero, el IAT es un instrumento autoinformado, lo que implica que 
los resultados pueden no ser del todo fiables, dado que los encuestados suelen tener una percepción errónea de sus propios comportamientos en Internet. Segundo, todas las muestras estaban compuestas de estudiantes universitarios, por lo que hay que tener cautela al generalizar los resultados a una población más diversa. Tercero, porque no se ha comprobado la invarianza factorial, las conclusiones sobre diferencias en países deben hacerse con cautela. Existe la posibilidad de que la estructura factorial del IAT pueda no ser comparable entre países porque no hay una estructura común.

Respecto del análisis comparativo del metaanálisis de Moon et al. (2018), no es nuestro objetivo analizar los procesos estadísticos asociados a cada estudio incluido aquí, por lo que algunas de las diferencias encontradas en o entre los países podrían atribuirse a los diferentes procedimientos estadísticos usados en vez de a la cultura.

También ha de tenerse en cuenta cómo el paso del tiempo puede haber afectado la efectividad del IAT para medir los problemas relacionados con el uso de Internet. El IAT fue desarrollado en 1998, antes del uso extensivo del Internet en el mundo. Estos últimos 22 años de desarrollo pueden haber afectado la relevancia del cuestionario y la importancia de algunos ítems. Por tanto, sería muy recomendable que el IAT, el cuestionario más popular en este campo, se actualizase para reflejar mejor los comportamientos en Internet que en la actualidad son populares y problemáticos.

\section{Conclusión}

Los hallazgos de nuestros tres estudios de análisis factoriales en EE. UU., España y Colombia mostraron resultados similares a los hallazgos anteriores en las mismas regiones. En EE. UU. encontramos tres factores: 1) Necesidad emocional (satisfacción de necesidades emocionales y dependencia); 2) Pérdida de control (incapacidad para controlar el uso y descuido de actividades importantes); y 3) Negligencia de las obligaciones (negligencia de las obligaciones a favor de Internet). En España también encontramos tres factores: 1) Pérdida de control (incapacidad para controlar el uso y negligencia de las obligaciones); 2) Necesidad emocional (satisfacción de necesidades emocionales); y 3) Dependencia. En Colombia encontramos 2 factores: 1) Necesidad emocional (satisfacción de necesidades emocionales y dependencia); y 2) Pérdida de control (incapacidad para controlar el uso y negligencia de las obligaciones). De los tres países estudiados, los participantes españoles tuvieron las puntuaciones más bajas en el IAT, coherente con un estudio anterior de Laconi et al. (2018).

Todos los análisis factoriales tenían un factor relacionado con problemas emocionales/psicológicos y otro factor relacionado con problemas de pérdida de control/gestión del tiempo, lo que sugiere que los problemas para controlar los impulsos y las necesidades emocionales insatisfechas son los componentes más importantes en el desarrollo del uso problemático de Internet en el mundo. Por tanto, recomendamos que los estudios sobre el uso problemático de Internet, que enfocan el Internet como una entidad causante de la adicción de manera similar a las drogas, se alejen del marco de la adicción, dirijan el foco hacia las motivaciones y las recompensas de los usuarios de Internet y desarrollen un nuevo concepto del uso problemático de Internet como la expresión de una interacción entre los problemas para controlar los impulsos y las necesidades emocionales insatisfechas en estos tiempos actuales tecnológicos.

\section{Conflicto de intereses}

Los autores declaran la inexistencia de conflicto de interés.

\section{Referencias}

Ahmad, M., Alzayyat, A. y Al-Gamal, E. (2015). The factor structure of the Internet Addiction Tool with university students in Jordan. Issues in Mental Health Nursing, 36, 725-731. doi:10.3109/01612840.2015.1033041.

American Psychiatric Association. (1994). Diagnostic and statistical manual of mental disorders (4th ed.). Washington, DC: Bell, C. C.

Bahrainian, S. A., Alizadeh, K. H., Raeisoon, M., Hashemi, G, O. y Khazaee, A. (2014). Relationship of Internet addiction with self-esteem and depression in university students. Journal of Preventive Medicine and Hygiene, 55, 86-89.

Barke, A., Nyenhuis, N. y Kroner-Herwig, B. (2012). The German version of the Internet Addiction Test: A validation study. Cyberpsychology, Behavior and Social Networking, 15, 534-542. doi:10.1089/cyber.2011.0616.

Bentler, P. M. (2006). EQS 6 structural equations program manual. Encino: Multivariate Software.

Bobes, B. M. T., Flórez, G., Seijo, P. y Bobes, G. J. (2019). Does ICD-11 improve the epidemiological and nosological purposes of mental, behavioral and developmental disorders? Adicciones, 31, 183-188. doi:10.20882/adicciones.1368.

Boysan, M., Kuss, D. J., Barut, Y., Ayköse, N., Güleçe, M. y Özdemir, O. (2017). Psychometric properties of the Turkish version of the Internet Addiction Test (IAT). Addictive Behaviors, 64, 247-252. doi:10.1016/j.addbeh.2015.09.002.

Bozoglan, B., Demirer, V. y Sahin, I. (2013). Loneliness, self-esteem, and life satisfaction as predictors of Internet addiction: A cross-sectional study among Turkish university students. Scandinavian Journal of Psychology, 54, 313319. doi:10.1111/sjop.12049.

Brown, T.A. (2006). Confirmatory factor analysis for applied research. New York: Guilford Press. 
Chen, Y. L. y Gau, S. S. F. (2016). Sleep problems and Internet addiction among children and adolescents: A longitudinal study. Journal of Sleep Research, 25, 458-465. doi:10.1111/jsr.12388.

Comisión Económica para América Latina y el Caribe. (2017). State of broadband in Latin America and the Caribbean: 2017. Recuperado de https://repositorio.cepal.org/bitstream/handle/11362/43670/1/S1800532_en.pdf.

Conti, M. A., Jardim, A.P., Hearst, N., Táki, A. C., Tavares, H. y Nabuco de Abreu, C. (2012). Evaluation of semantic equivalence and internal consistency of a Portuguese version of the Internet Addiction Test (IAT). Revista de Psiquiatria Clinica, 39, 106-110. doi:10.1590/S010160832012000300007.

Dhir, A., Chen, S. y Nieminen, M. (2015). Psychometric validation of Internet Addiction Test with Indian adolescents. Journal of Educational Computing Research, 53, 1531. doi:10.1177/0735633115597491.

Durkee, T., Kaess, M., Carli, V., Parzer, P., Wasserman, C., Floderus, B., Apter, A.,... Wasserman, D. (2012). Prevalence of pathological Internet use among adolescents in Europe: Demographic and social factors. Addiction, 107, 2210-2222. doi:10.1111/j.1360-0443.2012.03946.x.

Faraci, P., Craparo, G., Messina, R. y Severino, S. (2013). Internet Addiction Test (IAT): Which is thebest factorial solution? Journal of Medical Internet Research, 15, 220-230. doi: 10.2196/jmir.2935.

Fernández-Villa, T., Molina, A. J., García-Martín, M., Llorca, J., Delgado-Rodríguez, M. y Martín, V. (2015). Validation and psychometric analysis of the Internet Addiction Test in Spanish among college students. BMC Public Health, 15, 1-9. doi:10.1186/s12889-015-2281-5.

Fioravanti, G. y Casale, S. (2015). Evaluation of the psychometric properties of the Italian Internet Addiction Test. Cyberpsychology, Behavior, and Social Networking, 18, 120128. doi:10.1089/cyber.2014.0493.

Grande Gosende, A., Martínez Loredo, V. y Fernández Hermida, J. R. (2019). Gambling Motives Questionnaire validation in adolescents: Differences based on gambling severity and activities. Adicciones, 31, 212-220. doi:10.20882/adicciones.1057.

Guan, N. C., Isa S. M., Hashim, A. H., Pillai, S. K. y Harbajan Singh, M. K. (2015). Validity of the Malay version of the Internet Addiction Test: A study on a group of medical students in Malaysia. Asia-Pacific Journal of Public Health, 27, 2210-2219. doi:10.1177/1010539512447808.

Hawi, N. S. (2013). Arabic validation of the Internet Addiction Test. Cyberpsychology, Behavior, and Social Networking, 16, 200-204. doi:10.1089/cyber.2012.0426.

Hawi, N. S., Blachnio, A. y Przepiorka, A. (2015). Polish validation of the Internet Addiction Test. Computers in Human Behavior, 48, 548-553. doi:10.1016/j. chb.2015.01.058.
Ho, R. C., Zhang, M. W. B., Tsang, T.Y., Toh, A. H., Pan, F., Lu, Y., Cheng, C.,... Mak, K-K. (2014). The association between internet addiction and psychiatric co-morbidity: A meta-analysis. BMC Psychiatry, 14, 183. doi:10.1186/1471-244X-14-183.

Hofstede, G. (1983). National cultures in four dimensions: A research based theory of cultural differences among nations. International Studies of Management $\mathcal{E}$ Organization, 13, 46-74. doi:10.1080/00208825.1983.11656358.

Jelenchick, L. A., Becker, T. y Moreno, M. A. (2012). Assessing the psychometric properties of the Internet Addiction Test (IAT) in US college students. Psychiatry Research, 196, 296-301. doi:10.1016/j.psychres.2011.09.007.

Kardefelt-Winther, D. (2014). A conceptual and methodological critique of internet addiction research: Towards a model of compensatory internet use. Computers in Human Behavior, 31, 351-354. doi:10.1016/j. chb.2013.10.059.

Karim, A. K. M. R. y Nigar, N. (2014). The Internet Addiction Test: Assessing its psychometric properties in Bangladeshi culture. Asian Journal of Psychiatry, 10, 75-83. doi: 10.1016/j.ajp.2013.10.011.

Kaya, F., Delen, E. y Young, K. S. (2016). Psychometric properties of the Internet Addiction Test in Turkish. Journal of Behavioral Addictions, 5,130-134. doi:10.1556/2006.4.2015.042.

Korkeila, J., Kaarlas, S., Jääskeläinen, M., Vahlberg, T. y Taiminen, T. (2010). Attached to the web - harmful use of the Internet and its correlates. European Psychiatry, 25, 236-241. doi:10.1016/j.eurpsy.2009.02.008.

Laconi, S., Kaliszewska-Czeremska, K., Gnisci, A., Sergi, A., Barke, A., Jeromin, F., Groth, J.,... Kuss, D. J. (2018). Cross-cultural study of Problematic Internet Use in nine European countries, Computers in Human Behavior, 84, 430-440. doi:10.1016/j.chb.2018.03.020.

Laconi, S., Rodgers, R. F. y Chabrol, H. (2014). The measurement of Internet addiction: A critical review of existing scales and their psychometric properties. Computers in Human Behavior, 21, 190-202. doi:10.1016/j. chb.2014.09.026.

Lai, C. M., Mak, K. K., Watanabe, H., Ang, R. P., Pang, J. S. y Ho, R. C. (2013). Psychometric properties of the Internet addiction test in Chinese adolescents. Journal of Pediatric Psychology, 38, 794-807. doi:10.1093/jpepsy/ jst022.

Lee, B. W. y Stapinski, S. L. (2012). Seeking safety on the internet: Relationship between social anxiety and problematic internet use. Journal of Anxiety Disorders, 26, 197205. doi: 10.1016/j.janxdis.2011.11.001.

Lee, H. W., Choi, J-S., Shin, Y-C., Lee, J-Y., Jung, H. Y. y Kwon, J. S. (2012). Impulsivity in Internet Addiction: A Comparison with Pathological Gambling. Cyberpsychology, Behavior, and Social Networking, 15, 373-377. doi:10.1089/cyber.2012.0063. 
Lee, K., Lee, H-K., Gyeong H., Yu, B. y Kim, D. (2013). Reliability and validity of the Korean version of the Internet Addiction Test among college students. Journal of Korean Medical Science, 28, 763-768. doi:10.3346/ jkms.2013.28.5.763.

Lee, Y. S., Han, D. H., Kim, S. M. y Renshaw, P. F. (2015). Substance abuse precedes Internet addiction. Addictive Behaviors, 38, 2022-2025. doi:10.1016/j.addbeh.2012.12.024.

Lin, I-H., Ko, C-H., Chang, Y-P., Liu, T-L., Wang, P-W, Lin, H-C., Huang, M-F.,... Yen, C-F. (2014). The association between suicidality and Internet addiction and activities in Taiwanese adolescents. Comprehensive Psychiatry, 55, 504-510. doi:10.1016/j.comppsych.2013.11.012.

Lopez-Fernandez, O. (2015). Cross-cultural research on Internet addiction: A systematic review. International Archives of Addiction Research and Medicine, 1, 011. doi:10.23937/2474-3631/1510011.

Mohammadsalehi, N, Mohammadbeigi, A., Jadidi, R., Anbari, Z., Ghaderi, E. y Akbari, M. (2015). Psychometric properties of the Persian language version of Yang Internet Addiction Questionnaire: An explanatory factor analysis. International Journal of High Risk Behaviors and Addiction, 4, e21560. doi:10.5812/ijhrba.21560.

Moon, M. S., Hwang, J. S., Kim, J. Y., Shin, A. L., Bae, S. M. y Kim, J. W. (2018). Psychometric properties of the Internet Addiction Test: A systematic review and meta-analysis. Cyberpsychology, Behavior, and Social Networking, 21, 473-484. doi:10.1089/cyber.2018.0154.

Orsala, O., Orsalb, O., Unsalc, A. y Ozalp, S. S. (2013). Evaluation of Internet addiction and depression among university students. Procedia - Social and Behavioral Sciences, 82, 445-454. doi:10.1016/j.sbspro.2013.06.291.

Panayides, P. y Walker, M. J. (2012). Evaluation of the psychometric properties of the Internet Addiction Test (IAT) in a sample of Cypriot high school students: The Rasch Measurement Perspective. Europe's Journal of Psychology, 8, 327-351. doi:10.5964/ejop.v8i3.474.

Pedrero-Pérez, E. J., Ruiz-Sánchez de León, J. M., Rojo-Mota, G., Llanero-Luque, M., Pedrero-Aguilar, J., Morales-Alonso, S. y Puerta-García, C. (2018). Tecnologías de la Información y la Comunicación (TIC): Uso problemático de Internet, videojuegos, teléfonos móviles, mensajería instantánea y redes sociales mediante el MULTICAGE-TIC. Adicciones, 30, 19-32. doi:10.20882/ adicciones.806.

Pontes, H. M., Patrão, I, M. y Griffiths, M. D. (2014). Portuguese validation of the Internet Addiction Test: An empirical study. Journal of Behavioral Addictions, 3, 107-114. doi: 10.1556/JBA.3.2014.2.4.

Puerta-Cortés, D. X., Carbonell, X. y Chamarro, A. (2012). Analysis of the psychometric properties of the Spanish version of Internet Addiction Test. Trastornos Adictivos, 14, 99-104. doi:10.1016/S1575-0973(12)70052-1.
Samaha, M. y Hawi, N. S. (2016). Relationships among smartphone addiction, stress, academic performance, and satisfaction with life. Computers in Human Behavior, 57, 321-325. doi: 10.1016/j.chb.2015.12.045.

Sánchez-Carbonell, X., Beranuy, M., Castellana, M. y Chamarro, A. (2008). La adicción a Internet y al móvil. ¿Moda o trastorno? Adicciones, 20, 149-160. doi:10.20882/adicciones.279.

Seabra, L., Loureiro, M., Pereira, H., Monteiro, S., Afonso, R. M. y Esgalhado, G. (2017). Relationship between Internet addiction and self-esteem: Cross-cultural study in Portugal and Brazil. Interacting with Computers, 29, 767778. doi:10.1093/iwc/iwx011.

Servidio, R. (2017). Assessing the psychometric properties of the Internet Addiction Test: A study on a sample of Italian university students. Computers in Human Behavior, 68, 17-29. doi:10.1016/j.chb.2016.11.019.

Starcevic, V. (2010). Problematic Internet use: A distinct disorder, a manifestation of an underlying psychopathology, or a troublesome behaviour? World Psychiatry, 9, 9293. doi:10.1002/j.2051-5545.2010.tb00280.x.

Starcevic, V. (2013). Is Internet addiction a useful concept? Australian E New Zealand Journal of Psychiatry, 47, 16-19. doi:10.1177/0004867412461693.

Stevens, J. (2018). Internet Stats \& Facts for 2019. Hosting Facts. Recuperado de https://hostingfacts.com/internet-facts-stats/.

Sung, M., Shin, Y. M. y Cho, S. M. (2014). Factor structure of the Internet Addiction Scale and its associations with psychiatric symptoms for Korean adolescents. Community Mental Health Journal, 50, 612-618. doi:10.1007/s10597013-9689-0.

The Netherlands leads Europe in Internet access (2018). Recuperado de https://www.cbs.nl/en-gb/news/2018/05/ the-netherlands-leads-europe-in-internet-access.

Tsimtsiou, Z., Haidich, A. B., Kokkali, S., Dardavesis, T., Young, K. S. y Arvanitidou, M. (2014). Greek version of the Internet Addiction Test: A validation study. Psychiatric Quarterly, 85, 187-195. doi:10.1007/s11126-013-9282-2.

Tsitsika, A., Janikian, M., Schoenmakers, T. M., Tzavela, E. C., Ólafsson, K., Wójcik, S., Macarie, G. F.,... Richardson, C. (2014). Internet addictive behavior in adolescence: A cross-sectional study in seven European countries, Cyberpsychology, Behavior, and Social Networking, 17, 528-535. doi:10.1089/cyber.2013.0382.

Waqas, A., Farooq, F., Raza, M., Javed, S. T., Khan, S., Ghumman, M. E., Naveed, S. y Haddad, M. (2018). Validation of the Internet Addiction Test in students at a Pakistani medical and dental school. Psychiatric Quarterly, 89, 235247. doi:10.1007/s11126-017-9528-5.

Watters, C. A., Keefer, K. V., Kloosterman, P. H., Summerfeldt, L. J. y Parker, J. D. A. (2013). Examining the structure of the Internet Addiction Test in adolescents: 
A bifactor approach. Computers in Human Behavior, 29, 2294-2302. doi:10.1016/j.chb.2013.05.020.

Weinstein, A., Yaacov, Y., Manning, M., Danon, P. y Weizman, A. (2015). Internet Addiction and Attention Deficit Hyperactivity Disorder among schoolchildren. The Israel Medical Association Journal, 17, 731-734.

Widyanto, L. y Griffiths, M. (2006). "Internet Addiction": A critical review. International Journal of Mental Health and Addiction, 4, 31-51. doi:10.1007/s11469-006-9009-9.

Widyanto, L., Griffiths, M. D. y Brunsden, V. A. (2011). Psychometric comparison of the Internet Addiction Test, the Internet-related problem scale, and self-diagnosis. Cyberpsychology, Behavior, and Social Networking, 14, 141149. doi:10.1089/cyber.2010.0151.

Yao, M. Z. y Zhong, Z. (2014). Loneliness, social contacts and Internet addiction: A cross-lagged panel study. Computers in Human Behavior, 30, 164-170. doi:10.1016/j. chb.2013.08.007.

Younes, F., Halawi, G., Jabbour, H., El Osta, N., Karam, L., Hajj, A. y Khabbaz, L. R. (2016). Internet addiction and relationships with insomnia, anxiety, depression, stress and self-esteem in university students: A Cross-Sectional Designed Study. PLoS One, 11, e0161126. doi:10.1371/ journal.pone.0161126.

Young, K. (1998). Caught in the net. New York: John Wiley. 